\title{
Thoughts and facts about antibiotics: Where we are now and where we are heading
}

This article has been corrected since advance Online Publication, and a corrigendum is also printed in this issue.

\author{
János Bérdy
}

The declining trends in microbial metabolite and natural products research and the refocusing of this research area are discussed. Renewing natural products research requires inexhaustible natural resources, as well as new genetic techniques and microbial sources, including endophytic microbes. The numbers of known bioactive metabolites are summarized according to their microbiological origin, biological activities and chemical structures. Synthetic and natural product-based libraries are also compared. Importantly, the wide range of microbial metabolite bioactivities, future trends and the importance of prioritizing natural products over synthetic compounds are emphasized.

The Journal of Antibiotics (2012) 65, 385-395; doi:10.1038/ja.2012.27; published online 18 April 2012

Keywords: antibiotics; bioactive microbial metabolites; biodiversity; future prospects; non-antibiotic bioactivities; taxonomic diversity

\section{PREFACE}

The discovery and medicinal use of antibiotics in the 1950s have undoubtedly conferred one of the greatest benefits on mankind. Over the following 10-15 years, the average lifetime of the population increased significantly, some infectious diseases almost disappeared, and several neoplastic and viral diseases became controllable. Antibiotics were determined to be useful in the treatment of bacterial, fungal and protozoal infections and some physiological diseases (for example, lowering cholesterol). These compounds were also used in veterinary applications and as plant protecting agents (herbicides, pesticides) and feed additives. As a result, antibiotics were referred to as bioactive microbial metabolites.

In addition to the wonderful discoveries made over the past decades, antibiotics research has been full of scientific and, recently, economic challenges. Microbes continuously fight to survive, and although I have spent most my life searching for microbes and natural products, I am somewhat anxious for the future.

Scientists forget that during the million years of evolutionary development, microbes have acquired strong metabolic power. Because of their incredible adaptability, which requires a variety of biochemical mechanisms, microbes seem to have at least equal chances for survival as scientists devise methods to kill these organisms. This challenge is ongoing, and I hope that we will someday be able to control microbes.

The biggest advantage microbes have against antibiotics is their incredible adaptability to changes in their environment due to their flexible metabolic power. Antibiotic activity is only one, and perhaps not the most important, effect among the diverse biological, chemical and physical microbial activities. For millions of years, microorganisms have continuously interacted with their physical, chemical and biological environments. In addition, microbes interact with other living organisms, as studies have shown that these organisms communicate with each other (sociomicrobiology).

Most people ask the question, what went wrong? What do we have to do to prevent the survival of microbes? The answer is both everything and nothing. We must learn from the past to change the future. We have to leave behind the myopic feeling that we must understand everything about microbes.

This paper is based on my experience of more than half of a century, the data I have accumulated over time, and in some respects, my personal opinion.

\section{HISTORY OF THE LAST TWO DECADES (THE DECLINE AND REFOCUSING OF ANTIBIOTIC RESEARCH)}

The increasing resistance of antibiotics was the first problem derived from the widespread and uncontrolled application of antibiotics. Until the sixties, everything seemed to be well controlled. During this time, new natural sources of antibiotics, such as the rare actinos, and other non-medical applications increased. The number of new antibiotics, mainly analogs, increased almost exponentially. To eliminate antibiotic resistance, the pharmaceutical industry developed thousands of new semisynthetic antibiotics, opening up a new area of antibiotic discovery. ${ }^{1}$

After this 'golden age', from the sixties, medical and scientific fields, as well as the economic climate changed continuously, and by the nineties, these changes became significant. The efficiency of antibiotic 
research decreased, productivity of classical screening methods failed and rediscovery was more frequent. Only a few new chemical structures were discovered, and the majority of new compounds were 'variations of a theme.2,3

The technical improvements of isolation and dereplication methods increased the isolation of a number of new metabolites. ${ }^{4}$ New applications in non-medical areas were developed, but research costs continuously increased and less promising leads were discovered. The introduction of new compounds into therapy and the marketing of new products have significantly decreased from 20-30 new drugs per decade to 3-4 newly marketed drugs over the past years. The relative success of third-generation semisynthetic agents, the wide use of a few old and new compounds, such as daptomycin, represented no real breakthrough. ${ }^{5}$

More than $90 \%$ of the studies performed by large pharmaceutical companies between 1980 and 2003 resulted in decreasing profits due to increasing research expenses, the small number of new leads and regulatory obstacles. As a result, the government and almost all large pharmas moved away from short-term antibiotics to the profitable 'lifestyle' drug market, and those used to treat chronic diseases. Research efforts increased among academic institutions and mainly in small, new, specialized pharmaceutical/biotechnological companies, such as Ecopia and Cubist, with less financial resources but more scientific freedom. Today, the biggest problem is not the discovery of useful new antibiotics, but rather finding somebody who can develop them. After 1944, when the USA began producing penicillin, governments worldwide never took part in the production of antibiotics. ${ }^{6,7}$

\section{The decline of microbial metabolite research}

The declining trends in microbial metabolite research could be the result of a combination of the following reasons:

1. Human responsibility (irresponsible applications and underestimation of the microbes)

2. Scientific failure (limitations of some new high-throughput screening (HTS) methods and combinatorial synthesis)

3. Problematic economic-regulatory environment (high costs, licensing and strict regulations)

Human factors (anthropocentric view). People with access to the mass production and uncontrolled application of antibiotics have barely uncovered the microbial world. Resistance genes are already present in soil microbes. The use of high concentrations of a broad spectrum of antibiotics, uncontrolled agricultural applications and development of simple antibiotic resistance frequently resulted in many other unpredictable and dangerous consequences by inducing the full inherent defense mechanisms of microbes. Microorganisms replicate and mutate rapidly, exchanging their genetic materials, which results in a wide range of acquired resistance. The number of multiresistant pathogens is constantly increasing. Additionally, under the pressure of high levels of antibiotics, transcription and modulation, numerous undesirable acquired virulence characteristics represent one of the most serious dangers. The problem is derived from evading natural evolution. For example, the antibiotic production levels of industrial strains are sometimes magnitudes (that is, one billion times) higher than that of natural strains.

Today, more than $70 \%$ of pathogenic bacteria are resistant to most antibiotics on the market. Reappearing 'old' pathogens (mycobacteria) and new re-emerging opportunistic pathogens could potentially recreate the pre-antibiotic era. The mortality of some multiresistant infections has reached an alarming 50 to $80 \%$. In the United States, $\sim 2$ million people acquire bacterium infections in hospitals, resulting in close to 100000 deaths. Worldwide, over two million fatalities per year are due to bacterial infections. Close to two billion people carry Mycobacterium tuberculosis, and according to the WHO, by 2020, 35 million people will die from tuberculosis. Malaria and HIV account for 300 million illnesses and more than five million deaths each year.

Urbanization and global travel are intensifying the spread of bacterial infections. The aging of immunocompromised patients also increases the cost of treatment. The threat of bioterrorism is an awful reality. Many serious problems have been created by not only scientists, but by the entire human society.

Scientific failures. One reason for the declining trends in antibiotic research is the negative effect of several unrealized scientific expectations.

Over the past years, research efforts, namely the principles and techniques for obtaining new leads, have been refocused. Classical screening methods were changed by HTS methods and the extensive use of combinatorial chemistry. Unfortunately, both methods suffer from several shortcomings and have not resulted in substantial research improvements.

The main problem with one of the target-based automated HTS methods is that the potent inhibitors (the hits) are usually ineffective in the host. Additionally, there are low hit-to-lead ratios in both synthetic $(<0.001 \%)$ and natural products libraries. The new compounds are usually devoid of therapeutic activity due to difficulties in accessing the target, such as permeability and efflux. The discovered compounds are unable to cross the cell-wall of the target. Thus, combinatorial chemistry alone is insufficient for obtaining new leads in bioactive natural products research. However, it does have a complementary role, which is excellent for providing variations of natural product leads. The advantages of the natural product libraries exceed any random chemical libraries. Importantly, we have to understand that microbes are the best combinatorial chemists.

Economic and regulatory reasons. In addition to the previously discussed factors, increasing research costs and frequent misconceptions in the usage and marketing of antibiotics, there are other purely technical reasons, namely licensing and regulatory hurdles, that have a role in the withdrawal of large pharmas from antibiotic research.

The main patents of popular antibiotics have expired or will expire in the near future, and undesirable competition with generic drugs is a risk for large pharmas. The introduction of a new compound takes at least $10-12$ years and costs billions of dollars ( $\$ 800-2000$ millions per drug), and the lifetime of these drugs on the market is only $8-10$ years, providing additional fiscal challenges. ${ }^{8}$

Recently, new rigorous regulations for clinical trials and the safety of new therapeutics, such as chemotherapeutic drugs, were approved. Based on the current FDA regulations, streptomycin, natural tetracyclines and most aminoglycoside drugs would now be impossible to approve.

\section{Refocusing microbial metabolite research}

The reasons for the decline of microbial metabolite research have an important role in refocusing this area of research. To compensate for the relatively low productivity of classical screening, synthetic efforts are almost exponentially increasing. Currently, more papers are being 
published in the area of the synthesis of natural products (secondary metabolites), especially analogous compounds, than on the isolation and investigation of new metabolites. The volume of work is increasing in the areas of structure-activity relationship, rational drug design and computer-aided drug-design approaches, and templatebased total synthesis of antibiotic mimics. Combination chemistry was introduced into this field with great expectations, but its usefulness is questionable. These works have great academic significance. However, real practical success in this area, especially in the use of random chemical libraries, still remains to be realized. However, the synthetic modification of naturally occurring compounds into acceptable drugs has had great success, resulting in numerous drugs, such as new carbapenems, cephalosporins, azithromycin, tygecycline, daptomycin and telavancin/dalbavancin..$^{9-11}$

There have been interesting changes in the geographical distribution of research. New countries, mainly in Asia (China, Korea and Thailand), have become involved in developing antibiotics. However, in European countries, with the exception of the academic institutions in Germany, the discovery of new natural antibiotics has almost come to an end. In the last two years, Chinese laboratories have discovered close to $30 \%$ of all newly published microbial antibiotics. The discovery processes tend to be international, and $\sim 30-40 \%$ of research papers have originated from collaborative efforts involving researchers of other countries.

The two former leading countries, Japan and the United States, only have two-three multis and some new small companies. Japan and the United States are among the leading four countries, including China and Germany, conducting antibiotic research.

\section{ARE WE REVERTING BACK TO THE PRE-ANTIBIOTIC ERA?}

\section{How can we obtain new leads?}

The following questions are unavoidable: Is there a real public health crisis? How can we fix these problems? The answers are debatable. There are currently two main tasks to solve. To stop the decline of microbial metabolite research and increase the effectiveness of the discoveries, we must complete the following: ${ }^{12-17}$

1. Look for new natural resources.

2. Discover new principles, more selective and effective methods, and techniques to access the full, encrypted diversity of Nature.

3. Use several other less important methods.

There is an enormous amount of almost untouched natural resources available, and our knowledge in the field of microbial metabolism is constantly increasing. In addition to genomics, molecular biology, and proteomics, there are new techniques and branches of science, such as sociomicrobiology, nanotechnology, bioinformatics and new combined MS, NMR and crystallographic techniques. In addition to human intellect, we need excellent organization and cooperation and a good economic background. In my opinion, pure chemical approaches without the inspiration of Nature, with the exception of complementary roles, are less useful. I strongly believe that chemistry in this area, with the exception of template-based production of antibiotic derivatives, is only suitable for the modification of natural products.

Introducing new and advanced methods and principles for the identification of the hidden activity of microbial biosynthetic machinery is unavoidable. We have to revitalize the discovery of antibiotics. In addition to the discovery of new natural resources and the search for new and unique environmental surroundings, progress in molecular biology, chemical microbiology, genomics and advanced biotechnology (genetic engineering) is necessary. In addition, mining genomes and metagenomes for cryptic pathways, combinatorial biosynthesis and the intelligent modification of natural products are important. A better understanding of complicated drug-host interactions to discover new potential molecular receptors and to decode the whole human genome is the key for success. We have to understand the real role that secondary metabolites have in the community of microbes. This long-term endeavor requires patience and the close cooperation of government, industry and creative scientists both in academia and industry.

\section{New natural resources}

The aforementioned negative reasons do not hinder the development of antibiotic research alone.

As natural product resources are practically inexhaustible, the majority of the world's biodiversity remains to be explored. There are millions of prokaryotes and eukaryotes, such as plant, animal and microbial species, that exist in Nature, mainly in oceans and rainforests. However, only a small fraction of 250-300 000 living species have been investigated, identified and deposited. At least 1.5 million and nearly half a million fungal and higher plant species exist, respectively, in Nature. Millions of bacteria live in $1 \mathrm{~g}$ of soil. According to Krypides, $5 \times 10^{30}$ microbes live on Earth. The vast majority of known species have never been tested for any kind of bioactivity. Isolated compounds (natural products) from these species have either never or very rarely been tested for a wide range of bioactivities. Up to now, only $\sim 1 \%$ of existing actinobacteria (Actinomycetales) and not more than 5\% of known fungi are cultivable and can be identified using commercial methods. Of the millions of existing fungal species, only $\sim 25000$ have been identified. According to DNA analyses, the actual microbial diversity is much higher than expected. At least ten million microbial species (prokaryotes and eukaryotes) may exist in hidden (for example, symbionts and eukaryotes) or encrypted forms in Nature. ${ }^{18-22}$

In addition to common terrestrial sources (for example, soil samples), the other only partly explored reservoirs of microbes are oceans, higher plants and several environmentally unique sources.

Oceans are the largest source of microbes and new secondary microbial metabolites. Oceans occupy $70 \%$ of the Earth's surface with practically untouched fauna and flora and host $\sim 87 \%$ of life on earth. Marine sources, such as deep-sea sediments, from the seashore mud to the depths of 10000 meters are rich sources of microbes as soil samples. Several millions of undiscovered organisms, including microorganisms, are speculated to exist in oceans. A detailed investigation of the marine biosphere was only initiated in the last 15-20 years. Invertebrate marine animals, such as sponges, molluscs, ascidians, bryozoa, coelenterates and echinoderms, and/or their symbiotic-assemblaged fungi and bacteria are projected to lead to tens of thousands of new interesting compounds. According to conservative estimations, at least 30000 compounds, including many interesting new structures, have been isolated from marine organisms. ${ }^{23-26}$

Endophytic microorganisms, mainly fungi, have been coevoluting for millions of years with their eukaryotic hosts, the higher plants, representing an important increasing resource of new secondary metabolites. In the last decade, approximately half of newly discovered fungal metabolites (some 5000 compounds) were isolated from endophytic strains. ' $80 \%$ of endophytic fungi produce some kind of bioactive compound'. The world of higher plants, especially rainforests, has only been partly explored for the isolation of new compounds. ${ }^{27-30}$

Additionally, because of the microbial diversity driven by ecological and environmental forces, microbes living in unique surroundings 
and extreme environments, such as mine lakes and hyper-arid deserts, may also be promising starting points. Microbial biogeography and other factors have a role in increasing the biodiversity of strains.

To exploit these possibilities, more manpower is being invested into these areas. Currently, more laboratories are isolating new plant and marine products. In the past 10-12 years, more than 20000 and more than 30000 new marine-derived, and higher plant-derived compounds, respectively, were isolated. Only a few new structural types of promising lead compounds are included in the tremendous number of newly discovered plant-derived compounds. In contrast, several totally new chemical types of marine-derived compounds have been isolated. Many of these new compounds are structurally very similar to known microbial metabolites. Severals of them are already marketed or in clinical trials. This area is a promising field in natural products research.

In my opinion, nobody knows what fraction of secondary metabolites originally isolated from higher plants and marine invertebrates are really plant, animal or microbial products. For example, currently, more than 25 different endophytic fungal species have been reported to produce the classical plant product taxol (paclitaxel), which is one of the best-selling antitumor drugs. Taxol could be an antitumor microbial antibiotic. Very recently, ET-743 (Yondelis, Trabectedin), a complicated antitumor compound that has completed clinical trials and is isolated from a marine tunicate, was isolated from a symbiotic microbial (Endoecteinascidia) species. $^{31}$

Related to marine and plant-derived compounds are problems of endophytic-symbiotic systems, the interactions between two or more different organisms. The partners in these systems co-evolved during the evolutionary process. Both marine animals (host) and their assemblaged (symbiont) microbes and endophytic microorganisms living together with the higher plants represent an almost untouched reservoir of new bioactive metabolites with new bioactivities. These interactions are very complicated, and the details are less understood. The interactions may have defending, regulatory, nutritional and activating roles. In addition, these interactions are mutualistic when all partners benefit, or parasitic/pathogenic when one partner benefits at the expense of other. The oldest known example of symbiosis is lichens, which are assembled from fungi and algae.

Symbiotic interactions between insects, nematodes and bacteria and between humans and at least 1000 species of $10^{14}$ gut bacteria also exist. Bacteria on human skin have a similar role. The study of the human microbiome also requires increasing research efforts. The oldest known example of insect-actinobacteria symbiosis is the 1954 discovery of primycin, an approved drug (EBRIMYCIN). ${ }^{32}$

Bacteria and other microbes in Nature live in communities. The same genera and different phylotypes interact not only with each other but also with their environment. Symbiontic assemblages may be considered to be a new type of 'organ' in which well-organized inter- and intracommunication exists, such as quorum sensing. Secondary metabolites are the components that direct these interactions, representing the chemical interface between microbes, hosts and the environment.

Unfortunately, the industrial use of these endophytes, such as the production of taxol by fermentation, is still far from reality. However, bioprospecting of symbionts and endophytes offers promise. Horizontal gene transfer and/or genetic recombination between endophytes and hosts could occur. The cultivation of some sponge symbionts, which can sometimes amount to $50-60 \%$ of the mass of marine animals, seems to be a promising approach. In the future, I strongly believe that the symbiotic microbial population will produce a fraction of increasing numbers of promising higher plants and marine metabolites.

However, according to most opinions, the world's biodiversity is declining at an alarming rate. The uncontrolled destruction of the world's flora and fauna is occurring, as well as the destruction of rainforests, soiling and contamination of the oceans. The limitations on the sharing of biological resources across borders, unregulated trading and biopiracy are all dangerous factors hindering biodiversity.

\section{Genome mining}

Mining and engineering biosynthetic pathways. In addition to the exhaustive investigation of new marine organisms and higher plants, the manipulation of microbes represents an endless biosynthetic possibility, providing unlimited ways to reestablish microbes as a major source for new lead discovery. The biosynthetic potential of microbes is far from being exploited. The introduction of new advanced multidisciplinary approaches into research is an absolute requirement. The knowledge of general microbiology, genetics, molecular biology, biological chemistry and biotechnology, informatics and nanotechnology can be used to introduce many new methods and techniques to exploit hidden microbial diversity. Sleeping and cryptic biosynthetic pathways are present in all microbes and unculturable species, which are requisite factors for future microbial metabolite research. ${ }^{33,34}$

The recent advances in DNA sequencing coupled with bioinformatics have led to the easy determination of bacterial genome sequences. Today, thousands of complete bacterium genome sequences are known. The study of genomic sequence data deposited in databases proved that bacterial and other microbial genomes contain numerous (sometimes 20-25) independent pathways that direct the biosynthesis of different types of compounds. The mining of these sequences (silent genes) sometimes leads to absolutely new metabolites. . $^{35,36}$

Several strategies to increase the bacterial diversity include novel culturing methods, varying culturing environments (co-cultures or mixed cultures) and isolating rare or slowly growing microbes. Simple engineering of natural product biosynthesis using mutasynthesis (precursor-directed biosynthesis) has been proven to be very productive for obtaining unnatural natural products (hybrid molecules) in the area of macrolides, aminoglycosides, and glycopeptides. Unique new screening techniques that use new enzymatic methods to discover inhibitors of fatty acid biosynthesis have led to promising new lead compounds, such as platensimycin. Combinatorial biosynthetic approaches have been used for the direct fermentation of useful new drugs, such as epirubicin and erythromycin derivatives.

Microorganisms contain significantly more biosynthetic operon genes that are arranged in clusters than those involved in producing the isolated compounds. These genes usually remain undiscovered because we either screen strains only for a specific activity or new genes/pathways are unable to be activated under the conditions used, becoming dormant/sleeping genes. With the advent of recombinant DNA methods, numerous new techniques have been applied to activate the full biosynthetic capacity of microbes. Recombinant DNA techniques, heterologous DNA-based approaches, and the expression of foreign biosynthetic genes in an easily culturable heterologous host (E. coli) can provide unique compounds or scaffolds.

By using an organism-independent strategy, utilizing the total DNA isolated from environmental samples containing unculturable strains, exploiting their genes, clusters by cloning them into metagenomic DNA, we can express partial or complete biosynthetic pathways. This metagenomic approach seems to be the most promising. ${ }^{37,38}$ 
I believe that most, if not all microbes have hidden biosynthetic pathways that may lead to new metabolites and biological functions/ activities. Verifying the existence of these pathways helps to uncover the hidden life of microbes. The advances of modern physical methods (for example, MS and NMR) coupled with proteomics have enabled the identification of enzymes involved in the synthesis of secondary metabolites, leading to new ways to obtain new compounds. ${ }^{39,40}$

There is an almost endless list of new bioengineering and genetic methods that promise to access a number of new potential bioactive compounds, but the true potential of these approaches remains to be seen. In the past decade, more than $10 \%$ (in the last years, close to $25 \%$ ) of the newly discovered microbial metabolites were obtained by combinatorial biosynthetic, genetic, genomic and co-fermentation techniques. In the past, these methods were reduced to mutasynthetic methods, which were used to derive less than $5 \%$ of all metabolites.

\section{Other approaches}

Maximizing taxonomic diversity, the early dereplication of strains to find those worth being tested, may be helpful in different genera. The re-examination of old compounds and their rediscovery in other fields using new methods may sometimes lead to new useful compounds both in medical and non-medical areas (for example,, thiamulin, strobilurin and mycophenolic acid). Newly emerging target pathogens (for example, Helicobacter, Mycoplasma, Chlamydia and Eimeria species), new types of applications in agriculture (herbicides, pesticides and insecticides) and veterinary medicine (coccidiostatics, anthelminthics, antiparasitic agents and feed additives) may further widen the use of any newly discovered compound.

A potential source of bioactive compounds is the reinvestigation of metabolites previously deemed to be inactive, which represent $\sim 60 \%$ of known metabolites. The investigation of active compounds using new advanced techniques has been successful (for example, mycophenolic acid, Cellcept). Over the past decades, there have been hundreds of examples in which reinvestigating old, inactive compounds with modern advanced methods has been shown to have other useful activities. ${ }^{41}$

Detailed discussions of synthetic chemical methods have already been published in hundreds of publications. Personally, I am unsure about the success of this approach.

\section{THE PRESENT SITUATION}

\section{Numbers of known antibiotics and natural products}

The following important questions remain: what is the known and possible number of microbial metabolites and natural products, what types of producer species (Phyla, families and genera) exist? What chemical types and (bio)activities exist and will be accessible in the future?

Thanks to combinatorial chemistry, we currently know $\sim 8-10$ million synthetic chemical compounds. Most of these compounds are produced by the pharmaceutical industry. The number of published natural compounds derived from the living world may be close to half a million (with estimations between 300000 to 600000 ), including 60-80000 microbial metabolites (Table 1). Close to half of all known microbial metabolites (47\%) exhibit some kind of biological activity, whereas this number is lower in plant (7\%) and animal-derived (3\%) compounds.

The total number of marketed drugs used in human therapy is estimated to be $\sim 3500$ compounds, representing less than $0.01 \%$ of all known chemical compounds. The total number of drugs, including antibiotic and other therapeutics, that are 'direct' natural products, derivatives or mimics is at least 1300-1400 compounds, which is onethird of all the known marketed drugs. Approximately $50 \%$ of drugs are direct natural products or those based on natural product scaffolds. The distribution of naturally derived drugs is $\sim 0.1 \%$ of known natural products, representing a high success rate that is magnitudes higher than that observed for synthetic drugs. Of all the antimicrobial-antitumor drugs, natural product-derived drugs represent the majority, accounting for 50 to $70 \%$ of all naturally derived drugs. This percentage may be higher for antitumor drugs. Among synthetic drugs (synthetic antimicrobials), only quinolones and oxazolidinones have a role in the antimicrobial market.

The number of antibiotic drugs that are 'direct' natural products is $\sim 200-220$. The number of semisynthetic or modified natural product-based drugs is more than 250 , representing close to 500 of the microbially derived drugs. Currently, 50-100 new anti-infective natural products are in clinical trials. The percentage of natural antibiotic drugs among all known natural products is $\sim 0.5 \%$, and this rate is $1.6 \%$ for microbial products. Approximately half $(47 \%)$ of the microbial metabolites ( $\sim 33000$ compounds) exhibit some kind of biological activity, including antibiotic and 'other' effects, and $\sim 40 \%$ ( 28000 metabolites) are conventional antibiotics. Of course, there is some overlap among activities.

Unfortunately, a correct estimation of how many natural products may be isolated in the future is practically impossible. Some mathematical modeling predicts further, but not endless, increases. If not exponentially, this number will continue to increase. ${ }^{42,43}$

All statistical numbers listed in this paper are based on data derived from the Bioactive MICrobial Metabolite Database, which is a somewhat simplified, continuously updated version of the Bioactive Natural Products Database previously described ${ }^{44}$ and published in a

Table 1 Approximate number of known synthetic compounds, All natural and microbial products

\begin{tabular}{|c|c|c|c|c|c|c|c|c|}
\hline & \multicolumn{4}{|c|}{ Synthetic chemical compounds } & \multicolumn{2}{|c|}{ Natural products } & \multicolumn{2}{|r|}{ Microbial products } \\
\hline Numbers & \multicolumn{3}{|c|}{ 8-10 millions } & & \multicolumn{2}{|c|}{$\sim 500000$} & & $\sim 70000$ \\
\hline Drugs & \multicolumn{3}{|c|}{$2000-2500$} & & \multicolumn{2}{|c|}{$1200-1300$} & & $450-500$ \\
\hline \multirow[t]{2}{*}{ Percentage } & \multicolumn{3}{|c|}{0.005} & & \multicolumn{2}{|c|}{0.6} & & 1.6 \\
\hline & Natural product & $\%$ & Animal derived & $\%$ & Plant derived & $\%$ & Microbial derived & $\%$ \\
\hline \multicolumn{9}{|c|}{ Occurring bioactivities and drugs derived from natural products according to their origin } \\
\hline Total numbers & $\sim 500000$ & $100 \%$ & $\sim 100000$ & $\sim 20 \%$ & $\sim 350000$ & $\sim 70 \%$ & $\sim 70000$ & $\sim 10 \%$ \\
\hline Bioactives & $\sim 60000$ & $\sim 12 \%$ & $\sim 5000$ & $\sim 3 \%$ & $\sim 25000$ & $\sim 7 \%$ & $\sim 33000$ & $47 \%$ \\
\hline Drugs & $\sim 1300$ & $0.03 \%$ & $100-150$ & $0.001 \%$ & 600-1000 & $0.03 \%$ & $400-500$ & $0.6 \%$ \\
\hline
\end{tabular}


handbook. ${ }^{45}$ This handbook was widely distributed as a database version. ${ }^{46}$ The updated Bioactive MICrobial Metabolite Database has never been published in any form and is only the author's hobby. It currently (2011) covers more than 34000 bioactive microbial metabolites, and $98 \%$ of the entries have a known MW, $96 \%$ have known formulas and $\sim 90 \%$ have known chemical structures. This database covers $\sim 55000$ names (trivial, chemical, identical and trade names), 78000 different producer species with strain-collection numbers, and 250000 references, including patent numbers. Besides the physical, chemical, antimicrobial, antitumor, antiviral data, Bioactive MICrobial Metabolite Database covers hundreds of other known bioactivities that are related to the 16000 'antibiotic' compounds covered. Detailed information and answers to specific questions are available from the author.

\section{TAXONOMIC DIVERSITY}

\section{Distribution of producer species}

Table 2 lists the total numbers and distribution of natural products and those with bioactivity. Microbial metabolites and antibiotics isolated from microbes or higher organisms are summarized according to their main sources. The numbers and prospects of synthetic antibiotics and antibiotic derivatives are also included.

In the past 70 years, the efforts devoted to the discovery of new metabolites from different microbial sources have continuously changed. From the 50 s to 70 s, $\sim 60 \%$ of new antibiotics were isolated from actinobacteria, almost exclusively from the Streptomyces species. Over time, the ratio of 'rare actinomycetes' continuously increased from 5 to $\sim 30 \%$ by the 80 s. Currently, this ratio has decreased to $\sim 10 \%$. The significance of fungi as secondary metabolite producers, especially from the early 80 s, has continuously increased up to $55 \%$ due to the fascinating interest of endophytic fungi. Currently, the ratio of cyanobacterial products has reached from practically 0 to $10 \% .{ }^{47}$ The sum of other eubacteriales products decreased from $\sim 20$ to $10-12 \%$ (Table 3 ). The largest decrease occurred in actinobacterial products as their ratio decreased by $25 \%$. This decrease does not indicate that actinobacteria in the future would not represent a very

Table 2 Approximate numbers of known natural products (2010)

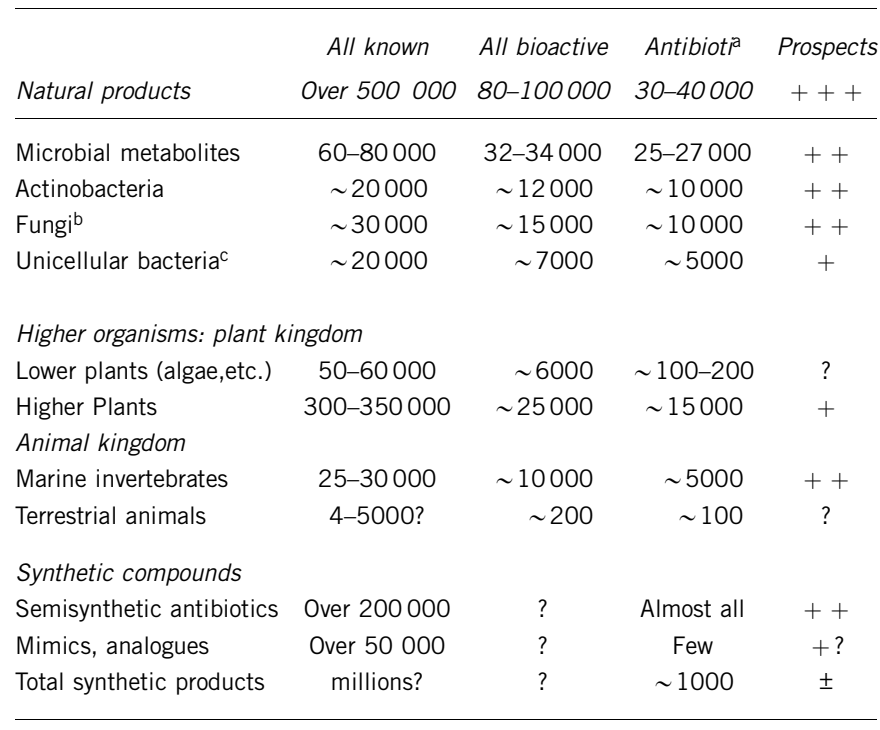

${ }^{a}$ Antinfective, antitumor and antiviral compounds.

${ }^{\mathrm{b}}$ Microscopic and basidimicetous fungi, myxomycetes, slime molds, yeasts.

cEukariotes including cyanobacteria and myxobacteria. important group of new secondary metabolite producers, but rather reflects a trend that frequently occurs throughout the history of antibiotic research. ${ }^{1,21}$

The production of bioactive metabolites in the microbial world is extremely unbalanced. Prokaryotic, unicellular bacteria (non-filamentous bacteria) and eukaryotic fungi produce practically all known bioactive microbial metabolites. In eukaryotic species, the filamentous actinobacteria, especially the historically important Streptomyces species, are common producers of bioactive compounds, producing $39 \%$ of all microbial metabolites. The Streptomycetales class containing the Streptomyces genus produces $\sim 80 \%$ of all metabolites. The other eubacteriales, such as unicellular bacteria, with the exception of the Bacillus and Pseudomonas species, seem to be relatively rare producers as $16 \%$ of all microbial products are derived from these organisms. Recently, among unicellular bacteria, cyanobacteria $(3.7 \%)$ and myxobacteria $(1.8 \%)$ species were found to be promising producers of microbial products with an increasing number of discovered compounds.

Today, the largest group of microbial metabolites is produced by various fungi $(45 \%)$, which include basidiomycetes (mushrooms; $11 \%$ ) and microscopic eukaryotic organisms (33\%), such as microscopic, filamentous fungi including Penicillium, Aspergillus and Trichoderma and hundreds of other species. These strains represent almost $99 \%$ of all fungal metabolites. Other types of fungi, such as yeasts and slime molds, are very poor producers, producing less than 400 products altogether, which is $\sim 1.5 \%$ of all metabolites.

In higher organisms, multicellular algae produce $\sim 3000$ natural products, including numerous bioactive compounds. From the diverse species of higher plants, an increasing number of new natural products have been discovered. The total number of plant-derived natural products is difficult to estimate but surely close to half a million. Approximately 100-150000 compounds exhibit biological and pharmacological activities, including some 20-25000 with chemotherapeutic effects. Marine invertebrates also produce a large number of antimicrobial and antitumor secondary metabolites that are estimated to be from 15000 to 35000 . The lichens (symbionts of algae and fungi), animal unicellular protozoa, lower plants (ferns), and terrestrial invertebrates and vertebrates from the Animal Kingdom (insects, worms, amphibians, and mammals) produce a lower amount of several hundreds to thousands of less significant bioactive metabolites. Generally, secondary products from higher forms of life show less variability and specificity compared with microbial products. Among higher plant products, the terpenoids, alkaloids and flavonoids, which have less chemical and biodiversity, mainly dominate.

The biosynthetic specificity of microbial species and genera is interesting because biosynthetic pathways are unevenly distributed across the large groups, and several distinct types of producers almost exclusively produce some structural classes of natural products. The following classes of compounds are produced almost exclusively by members of the Actinomycetales family: polyene macrolides, other large-membered macrolides (oligomycin-type), anthracyclines (daunomycin-type), polyether antibiotics (nigericin-type), cyclopolylactones (nonactin-type), most aminoglycosides, and anthracyclines, streptothricins, actinomycins and quinoxaline-peptides. Glycopeptides and orthosomycins are mainly produced by non-Streptomyces actinobacteria. Small-membered ring lactones (10-14), benzoquinone derivatives, gliotoxin-like diketopiperazines, peptaibols, grisans, scirpenes, cytocalasins, several distinct types of cyclopeptides (cyclosporins), alpha pyrones, numerous types of terpene types (generally polyketide synthase-derived compounds) are mainly fungal products. 
Table 3 Approximate number of bioactive microbial metabolites in periods from 1940 to 2010 according to their producers

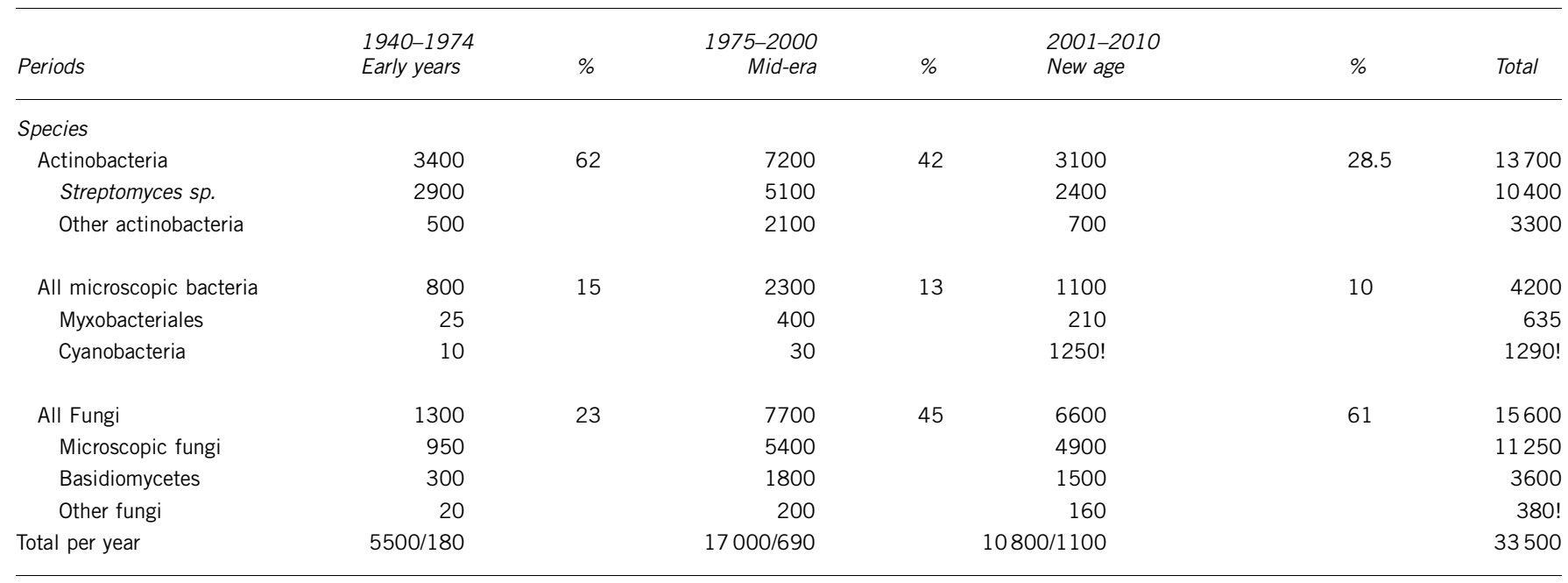

Lantibiotics and numerous types of lipocyclopeptides are almost exclusively unicellular bacterial products; however, these microbes never produced macrolides.

The biosynthetic capability and specificity of the thousands and millions of potential and existing microbial species in Nature remain to be understood. Only a relatively small number of the following genera and species, and a definite number of similar fungal species representing only the minority of the known microbes produce $\sim 80 \%$ of all known secondary metabolites: actino-, cyano-and myxobacteria, Bacillus, Pseudomonas, Penicillium and Aspergillus. Several common natural products, such as terpenoids and flavonoids, are very rarely produced by actinobacteria, but the relatively large molecular macrocyclic lactones, lactams, specific cyclopeptides, depsipeptides and polycyclic quinone-based molecules, and other unique and structurally complex compounds are almost exclusively produced by actinobacteria. In general, fungi produce relatively large numbers of low-MW compounds that are mainly polyketide synthase-derived. Unicellular bacteria produce mainly different kinds of peptides. Among the proteobacteria-derived compounds pseudomonic acid/ mupirocin (BACTROBAN) interestingly is a marketed drug. ${ }^{4}$

Two-thirds (68\%) of all cyanobacterial products (over 1200) are ribosomal-derived peptides, mainly large cyclopeptides and peptolides, with MWs between 800 and 1200. Most of these compounds exhibit various enzyme (proteases) inhibitory activities. Dolastatin, originally isolated from a marine mollusc, is actually a cyanobacterial product currently in clinical trials. One-third (32\%) of the 650 known myxobacterial products are macrolactones and macrodiolides, which are sometimes combined with heterocyclic rings, including epothilones that are also in clinical trials. ${ }^{47}$

In the distribution of the known bioactivity of microbial metabolites derived from different microbes there are only a few interesting results. More than $85 \%$ of all known bioactive microbial metabolites exhibit antibacterial, antifungal or antiprotozoal activities. Approximately 8600 compounds (26\%) exhibit exclusive or additional antitumor/cytotoxic activity, and $\sim 1200$ metabolites (4\%) display antiviral activity. From the more than 16000 fungal metabolites close to 10000 metabolites (66\%) exhibit non-antibiotic, mainly phytotoxic, plant growth regulating activities. Compounds with 'other' bioactivities are produced by $40 \%$ of actinobacteria, almost $70 \%$ of cyanobacterial species, and only $23 \%$ of other microscopic bacteria.
Currently, more than 25000 distinct microbial species, including bacteria, actinobacteria and fungi, have been reported to be producers of bioactive microbial metabolites. A compound may frequently be produced by multiple (sometimes 20-25) organisms that belong to a different genus, family, class or phylum. In addition, a specific microorganism can produce more (sometimes 15-20) metabolites, sometimes with different chemical types. My laboratory may have set the record in this field in 1977 when we isolated exactly 50 individual compounds from a fermentation broth of an industrial Micromonospora species. However, these compounds were close analogs and 'minor components' of this given strain. $^{48}$

\section{BIODIVERSITY}

\section{Non-antibiotic bioactivities}

Most microbial metabolites exhibit many biological activities. However, these activities are not necessarily essential for the producer species, do not have direct effects on the producers, and have no definite role in the life cycle of microbes. Then, why are they produced at all?

In general, the main effects of secondary metabolites are defense, regulation and communication. In addition, their chemical and physiological functions are diverse. They may function as vitamins, cofactors, information storage devices, hormones, pheromones, odorants, neurotransmitters, colorants/dyes, wound-healings, toxins, antineoplastics, pharmacologically active agents and antibiotics. In addition, their inherent biological-cellular function is extremely diverse. For example, they may modulate cellular transcription, multiplication, gene transfer, phage induction, host-cell response activity, inter and intracellular signaling, and induce resistance and virulence. The variations of the possible effects and activities of these compounds are practically endless.

For thousands of years, over the course of human history, various remedies derived from plants, animals and macroscopic fungi were used for medicinal purposes. Why did the antibiotic effect become the most popular? Why were antibiotics the first compounds to be discovered and produced on a large scale? The year 1929 was a key year in microbial metabolite research for medicinal purposes because of Fleming's discovery. However, I personally consider 1940-42 as the beginning of the real antibiotic era. This was a unique 
time as the following events made the incredible successes of antibiotics possible:

i The pressure of the 2nd World War.

ii The demand for chemotherapy due to Ehrlich's discoveries.

iii Simple in vitro detection.

During the 2nd World War, in the US, the cooperation of European scientists quickly led to the industrial production of penicillin, discoveries of othe antibacterial compounds (streptomycin, tetracyclines) and coining of the term antibiotic.

The aim of this review is to provide a detailed analysis of antimicrobial, antitumor, antiviral and agricultural applications of antibiotics, their effects and antibiosis. Hundreds of excellent reviews are available on this topic. Thus, I will focus on the so-called nonantibiotic and 'other' effects of both antibiotics and non-antibiotic secondary microbial metabolites.

An important study was conducted in the late 60s (1969) by Hamao Umezawa, ${ }^{49}$ who investigated the non-antibiotic bioactivities of antibiotics, namely their enzyme inhibitory activities. Years later, several hundreds of the most variable types of 'other' activities (for example, pharmacological, agricultural, biochemical, regulatory, metabolic and other biophysical activities) of microbial metabolites were discovered. Less than one-half of the known 'antibiotics' exclusively exhibit or have been identified as having antibiotic effects. Microbial compounds with only antitumor or antiviral activities are strictly chemotherapeutic agents and not antibiotics.

I think it is the time to step away from the term 'antibiotic'. However, it is clear to me that over the years, this term has been embedded in everybody's mind.

Up to mid-2011, almost 30000 different types of natural compounds with 'other' bioactivities were published, representing more than 16000 microbial compounds. The most frequently identified biological effects that represent 'other' activity were enzyme inhibitory activities both during the screening and investigation of isolated metabolites. Today, the enzyme inhibitory activities of $\sim 4500$ microbial compounds are known. Over the past years, hundreds of enzyme systems were investigated, most frequently various peptidases, proteinases, glycosidases, amylases, HIV integrases, protein kinases, acetyl-coenzyme A acyltransferase (ACAT), phosphatases, squalene synthetases, 3-hydroxy3-methylglutaryl-coenzyme A reductases, beta-lactamases and monoamine oxidase (MAO). Additionally, more than 3000 pesticides, antiparasitic and herbicide activities, including phytotoxic, plant growth regulatory, insecticide, nematocide, miticide, larvicide, anthelminthic, acaricide, ichthyotoxic, algicide and amoebicide activities, were reported. More than 2000 various pharmacologically active compounds with anti-inflammatory, neuroactive, antidepressants, anti-Alzheimer, cardioactive, cholesterol lowering, platelet aggregation inhibitors, antioxidants, hypotensive and vasodilatory agents, nerve growth factor (NGF), interleukin and endothelin antagonists, estrogens, anti-ulcer and antiallergic, and antihistamine effects were added to this group. Approximately 1500 immunoactive, -suppressants, -modulators and -stimulators, as well as compounds that have angiogenesis, proteasome and tubulin polymerase inhibitory effects, toxic actions, mitotic activities and apoptosis-inducing effects, have a role in anticancer chemotherapy. In addition to regulatory agents, the following are the other more frequently occurring effects and activities of microbial metabolites: antimetabolites, various stimulatory compounds, calcium antagonists, chelate-forming agents, siderophores, morphogenic agents, signaling/quorum-sensing compounds, scavengers, biosurfactants, feed additives, microbial hormones and preservatives.
Today, more than half $(16800 / 33000,51 \%)$ of all known 'antibiotics' exhibit other bioactivities. The percentages of fungal, actinobacterial and microscopic bacterial products in this group are $57 \%$, $38 \%$ and $8 \%$, respectively. Actinobacterial products (more than $70 \%$ ) mainly exhibit antimicrobial activity. More than $50 \%$ of fungal metabolites exhibit phytotoxic and herbicidal/pesticidal activities. However, only $2 \%$ of actinobacterial products exhibit phytotoxic activity. Most actinobacterial metabolites are enzyme inhibitors.

The fungal-derived cyclosporine (SANDIMMUNE) and statins (MEVACOR and ZOCOR) occupy a great fraction of the immunosuppressive and hypocholesterolemic market, respectively. In addition, tacrolimus (PROGRAF), rapamycin (RAPAMUNE) and spynosins are widely used in agriculture.

These bioactivities share a high degree of overlap, not only with antibiotic activities but also with each other, as illustrated in Figure 1. The most trivial overlapping exists between the enzyme inhibitory activities and other pharmacological and agricultural activities. Some metabolites exhibit three, four or more bioactivities. There are several small-molecular fungal macrolactones, such as brefeldin, which are isolated from various sources and possess more than 15 distinct bioactivities. Several older antibiotics, such as erythromycin, have other medically useful effects, such as gastro-motility inhibition. There are also many compounds with non-antibiotic effects that lack any antimicrobial activity, such as destruxin.

The aforementioned list of bioactivities is far from complete, but the observed bioactivities cover all areas of human pharmacology and agriculture, demonstrating the significance of efforts in this field. Unfortunately, until now, we have not had enough information on all aspects of the microbial physiology, evolutionary biology and ecology of these low-MW compounds.

\section{General considerations: the real role of antibiotics in nature} Analysis of the diverse types of natural molecules, their specific building blocks, possible active sites and producer species by intelligent comparison with their observed bioactivities has demonstrated numerous inherent consequences about the real biosynthetic mechanisms and the microbial life process, and structure-activity relationships. For example, we can understand the chemical structures (sometimes very simple compounds) that frequently exhibit analgetic and/or anti-Alzheimer effects.

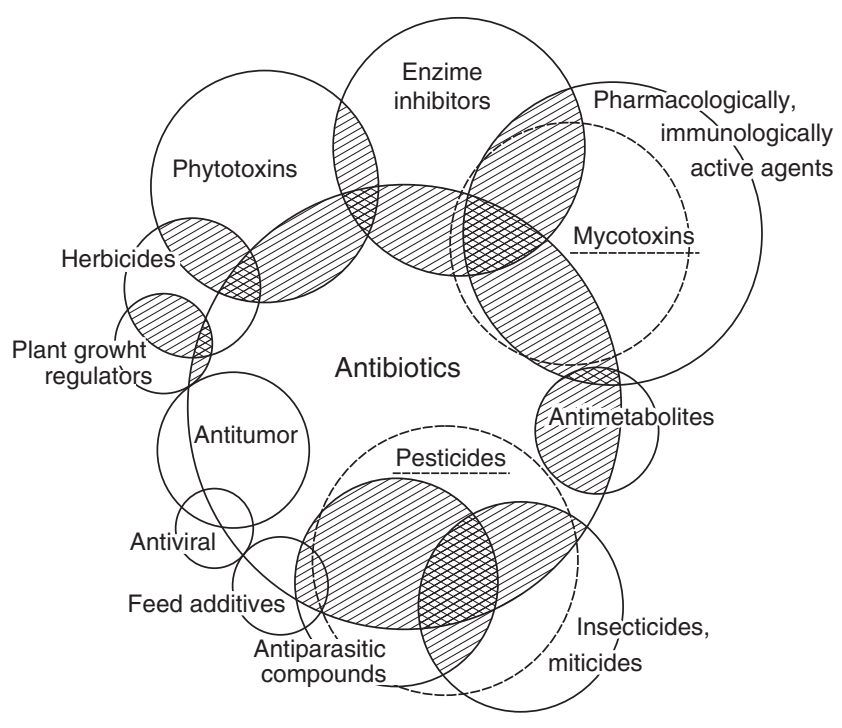

Figure 1 Bioactive microbial metabolites. 
In general, several facts complicate the analysis as bioactivities are displayed on a molecular level, using diverse interactions with various targets under variable conditions. For example, at different subinhibitory concentrations, different activities (for example, mutation inducing activity or hormesis) may occur. The chemical profile of metabolites produced in laboratories, especially by industrial strains, may be quite different due to numerous artificial mutations compared with strains growing in the environment. There is still no explanation as to why antibiotic production is restricted to several distinct species.

There are more complicated connections, interactions and conditions that exist in Nature than predicted. As Hamlet said, 'There are more things in heaven and earth, Horatio, than are dreamt of in your philosophy.

In fact, microbial metabolites serve as the chemical interface between the microbial world and the rest of Nature (for example, other living organisms and the environment). These compounds are intermicrobial and general signaling agents. The clear implication of their role helps to provide a better understanding of the real ecological function of microorganisms in the biosphere. ${ }^{50-52}$

Different types of microbial secondary metabolites may possibly have some kind of inherent feature that justifies their production and existence in Nature. These metabolites may express some type of undiscovered chemical or biological activity. Numerous data reflect the very complicated, diverse and sometimes unknown microbial biosynthetic machinery, especially in the case of unculturable species. There is no reason to propose that newly studied microbes exhibit numerous, unknown building blocks and new machinery to assemble these blocks, forming unimaginable new structures with potentially new novel bioactivities. The real task is to access these new organisms, building blocks and biosynthetic machineries. When this enormous task is completed, we will have access to an almost endless number of new, useful compounds for the benefit of humankind.

\section{CHEMICAL DIVERSITY}

The complicated structures of microbial secondary metabolites cover almost all functional groups in organic chemistry and are frequently inaccessible by synthetic libraries and other synthetic methods. The molecular weights of microbial metabolites vary between 70 (acrylamidine), 2539 (ramoplanin), 3354 (nisin), 7457 (microcin-24), to as high as 12524 (actinohivin). The average MW values are between 400 and 600 . The average MW of actinobacterial products is $\sim 550$, whereas fungal products have an average MW of $\sim 380$. Generally, actinobacterial products have more complicated structures than most fungal metabolites, reflecting their somewhat different biosynthetic machinery. ${ }^{53,54}$

Over the past 30 years, no breakthrough novelties or totally new large group of antibiotics have been discovered. However, extremely large numbers and variations of unique, almost unimaginable structures were discovered, consisting of a surprising combination of known moieties. This diversity may be comparable to that of the biological activities of metabolites. An illustration of only a few fascinating new structures would occupy far too much space in this paper. Readers are familiar with the topic and are well aware of the fascinating chemical structures that have been published in the past decade(s). These compounds can be found in several public databases (Antibase, Chapmann and Hall database, CRC Handbooks, etc.) and recent papers published in the leading journals of this field (the present Journal, the J. Natural Products) and in leading chemical journals of ACS and other publishers. The chemical structure of one of the recently discovered promising actinobacteria derived metabolite, kibdelomycin (Figure 2), illustrates these new structural arrangements. ${ }^{55}$
The structural features are distinctly unparalleled with synthetic and natural product drugs (see previous sections). Naturally derived drugs, such as the microbial natural products, have high steric complexity with many chiral centers. These compounds have less nitrogen and more oxygen content, as well as less aromatic and more ether linkages. Generally, these compounds are more polar and have specific structural features (for example, up to 60-membered macrolactone rings and cyclic peptide structures), which rarely occur among common synthetic compounds.

The biosynthetic capacity of microbes, especially actinobacteria, is also inexhaustible. In addition to the basic pathways (for example, polyketide synthase), the microbes are able to produce new types of compounds via extensive branching, and a series of alternative reactions by different enzymes, condensations, alkylations, oxidations, isomerizations and simple decoration. The activation of hidden/silent microbial genes, as well as new variations and skeletons of compounds are likely to be discovered in the future. ${ }^{56}$

\section{THE FUTURE}

Chemical, biotechnological and combination approaches are used to find new leads for new chemotherapeutical agents, antibiotics and other microbial drugs.

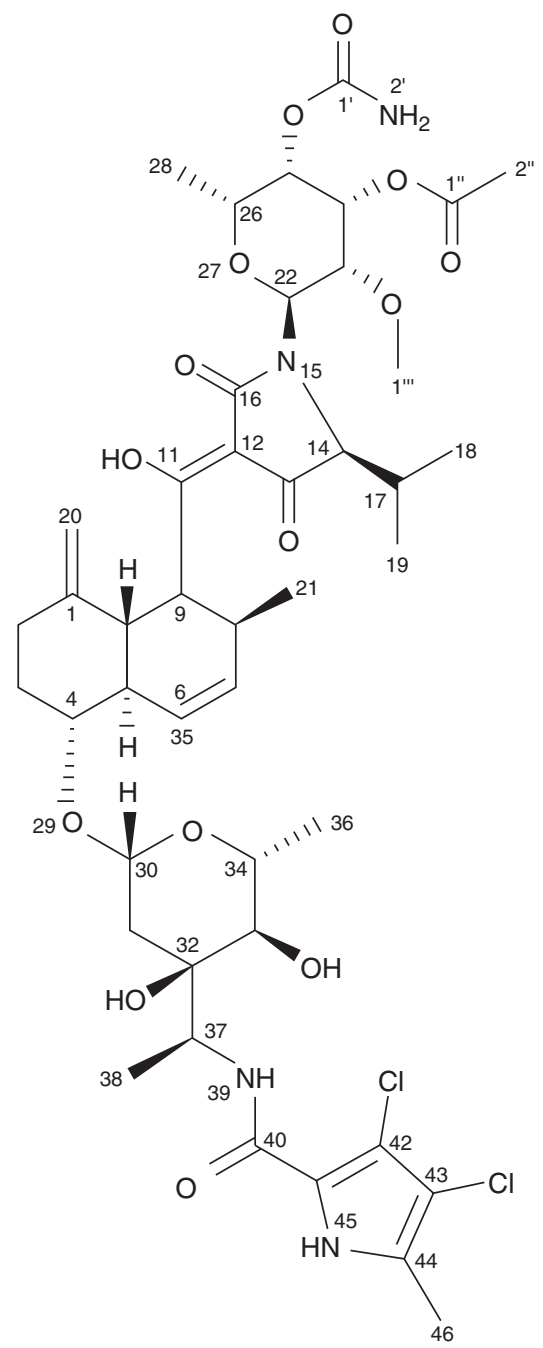

Figure 2 Kibdelomycin. 
Which approach is the best to obtain new bioactive (anti-infective or antitumor) microbial drugs in the future? The following approaches are the main routes to obtain new leads for developing new antibiotic drugs:

1. Chemical/synthetic approach (rational drug design, structure activity relationship, combination synthesis, template-based or random total synthesis)

2. Biotechnological/fermentation approach (using classical or genetic methods and diversification of natural products libraries to produce new natural products)

3. Combination/semisynthetic approach (modification of known compounds by chemical or genomic methods)

All of these approaches have their advantages and disadvantages.

1. Advantages: An endless number of synthetic products and combinatorial libraries will be accessible. Several groups and ring systems that are missing in Nature and usually not requested for bioactivity will be available. Additionally, reactions can easily be scaled-up without having to perform purification steps.

Disadvantages: There is no way to predict how effective these synthetic products are in addition to their safety, uptake and access to the target, typically a single target. Template-based synthesis, structure activity relationship or rational drug design approaches have rarely led to practical results. Combinatorial chemistry itself is insufficient, but it may have a complementary role in the modification of natural products.

2. The advantages of the natural products are their structural diversity, steric complexity, multiple chiral centers, as well as their high potency, selectivity and multiple modes of action. Because of their multicenter ligand-protein interactions, natural products provide functional diversity and selectivity for more than one target. Their host compatibility, drug-like structures and good correlation with human targets guarantee diverse in vivo effects. The possible 'cross-talk' between microbes via their metabolites and host cells led to the discovery of numerous antitumor and antiviral agents. Microbial metabolites act on enzyme families and have various effects on the immune system, which has led to their use in a number of other therapeutic areas. Statins and cyclosporines are among the world's best-selling anticholesterolemic and immunosuppressive drugs. There are cases of a single metabolite, such as brefeldin, exhibiting 10-15 different bioactivities due to having multiple binding sites on host cells. ${ }^{57-59}$

Finally, natural products generally meet the requirements of green chemistry.

The disadvantages of natural products are that a well-trained team of scientists from different disciplines and specific equipment are required. In addition, the scale-up of natural products is problematic. Natural product extracts are always complicated mixtures, making the isolation of compounds challenging.

3. The natural products are preeminent leads for successful structural modifications to increase or change the therapeutic activity of isolated compounds by combining the advantages of biotechnological and chemical approaches. Real success can only be achieved using biotechnological and semisynthetic approaches or their logical combination. In the last decade, engineering and mining the biosynthetic genes, the diversification of natural product libraries and modification by chemical or genetic methods of known natural antibiotics have been the most effective ways to find new chemotherapeutic agents. Natural products have many inherent favorable properties. Sometimes improved solubility, stability and absorption properties require only minor modifications of the core structure Substitution and decoration with several unnatural moieties can increase the stability, solubility, spectrum broadening and activity of natural products against resistant strains and provide more favorable pharmacodynamic properties. My analysis begins with the making of semisynthetic penicillins and culminates with natural product derivatives, such as tigecycline, dalbavancin and telavancin. The only disadvantage of these approaches may be increasing costs.

I believe that natural products, including those with chemical and genetic modifications, are compounds that will be successful therapeutics in the future. The most prescribed anti-infective drugs in developing countries are semisynthetic or modified antibiotics. Based on numbers and percentages, we can easily conclude that the future of antibiotic discoveries depends on our understanding of Nature, especially of microorganisms. 'Nature is the best, most sophisticated, versatile and energetic combinatorial chemist'. The creativity of Nature is far beyond the imaginations of people, including chemists. How could organic chemists from 1944 or 1929 think of a way to successfully synthesize penicillin? However, fungi can do it. Fleming only recognized the activity of penicillin, hundreds of scientists identified the structure, and the antibiotic was produced under the pressure of the war. As an organic chemist, I have no idea how long it would take for a synthetic chemist to invent penicillin without the inspiration of Nature.

In the future, I am sure that the discovery of new types of organisms, biosynthetic methods and clarified microbial biosynthetic machinery will lead to discovery of new bioactivities and types of structures. The mostly undiscovered world of the microbial communities surely will provide us with several pleasant surprises.

Lastly, a frequently forgotten problem is drug-host interactions. This is frequently the dividing line between the success of natural and synthetic products. A new compound is easy to synthesize, but to obtain new pharmacologically active compounds is challenging. To find suitable molecules that meet all of the complicated requirements, including bacterial targets and the specific features of the host (the human) is one of the most complicated tasks. To act effectively on thousands of pathogens and viruses, and effectively cure neoplastic diseases requires access to drugs that interact with these pathogens or cells, are transferred across membrane(s) and bind to target(s). Drugtarget-host interactions are always recurring problems. In addition to having in vitro and some in vivo activity, a marketable drug has to fit the following three requirements: accessibility, specificity and low toxicity.

Uptake and transport: Drugs must be either absorbed orally or by i.m. or i.v. injection, which requires suitable solubility, access to the desired pathogen or organ and effective penetration into the outer barriers of cells (on different membranes). Bioavailability: Drugs have to bind to the prosthetic group $(s)$ of the target. The target advantageously is a valid, non-hypothetical (as in HTS), with multiple binding sites (enzyme systems). Drugs have to be specific enough without binding to other organs, act specifically on the target without any undesirable side effects or toxicity and be stable and resistant against the environment in the host. Most problems with synthetic and HTS-derived compounds have been the lack of most of these requirements.

This is an interesting feature of bacterial life that has a lot of implications for humans. The biggest advantage of natural or naturally derived compounds is the existence of a basic similarity between the enzyme and other systems of evolutionally developed producers and hosts. Thus, these compounds are preeminent drug leads. 


\section{EPILOGUE}

Currently, 25-30000 various, clinically described human, mainly 'physiological' diseases have been identified. We can treat most of them symptomatically but can only effectively cure a small number of these diseases. Additionally, at least thousands of bacteria, fungi and parasites can cause life-threatening infectious diseases. The biggest problem between the increasing resistance and virulence of known pathogens is reemerging tuberculosis, HIV and malaria. The question is whether the present pharmaceutical and chemotherapeutical armamentarium is efficient at reducing these dangers? The answer is no. Many new useful drugs are required for chemotherapy and agriculture to continuously cure and feed an increasing population. Careful reconsideration of mediocre decisions is necessary. Scientists are heading in the right direction with respect to their way of thinking, and the minds of the other participants (the multis) in this research will change sooner or later.

\section{ACKNOWLEDGEMENTS}

I would like to express my appreciation to my colleagues all over the world, who have helped me by sending me their files and other information. In addition, I would like to express my deepest gratitude to my daughter, Ildiko, and granddaughter, Dora, for their valuable technical assistance.

1 Bérdy, J. Bioactive microbial metabolites. A personal view. J. Antibiot. 58, 1-26 (2005).

2 Demain, A. L. \& Sanchez, S. Microbial drug discovery: 80 years of progress. J. Antibiot. 62, 5-16 (2009) and references cited therein.

3 Fernandes, P. Antibacterial discovery and development - the failure of success? Nat. Biotechnol. 24, 1497-1503 (2006)

4 Butler, M. S. \& Cooper, M. Antibiotics in the clinical pipeline in 2011. J. Antibiot. 64 , 413-425 (2011).

5 Donadio, S. et al. Antibiotic discovery in the twenty-first century: current trends and future perspectives. J. Antibiot. 63, 423-430 (2010)

6 Hancock, R. E. The end of an era? Nat. Rev. Drug Discov, 6, 26-29 (2007).

7 Payne, D. J., Gwynn, M. N., Holmes, D. J. \& Pompliano, D. L. Drugs for bad bugs: confronting the challenges of antibacterial discovery. Nat. Rev. Drug Discov. 6, 29-40 (2007).

8 Katz, M. L., Müller, L. V., Polyakov, M. \& Weinstock, S. F. Where all the antibiotic patents gone? Nat. Biotechnol. 24, 1529-1531 (2006).

9 Davies, J. How to discover new antibiotics: harvesting the parvome. Curr. Opin. Chem. Biol. 15, 5-10 (2011).

10 Cordell, G. A. \& Colvard, M. D. Natural products in a world out-of-balance. Arkivoc VII, 97-115 (2007).

11 von Nussbaum, F., Brands, M., Hinzen, B., Weigand, S. \& Häbich, D. Antibacterial natural products in medicinal chemistry - Exodus or revival? Angew. Chem. Int. Ed. 45, 5072-5129 (2006).

12 Nicolaou, K. C., Chen, J. S., Edmonds, D. J. \& Estrada, A. A. Recent advances in the chemistry and biology of naturally occurring antibiotics. Angew. Chem. Int. Ed. 48, 660-719 (2009).

13 Anonymous. Bring the magic back to the bullets. Nat. Biotechnol. Editorial 24, 1489-1495 (2006)

14 Baltz, R. H. Antibiotic discovery from actinomycetes: will a renaissance follow the decline and fall? SIM News 55, 186-196 (2005).

15 Baltz, R. H. Antimicrobials from Actinomycetes: back to the future. Microbe 2, 125-133 (2007).

16 Baltz, R. H. Renaissance in antibacterial discovery from actinomycetes. Curr. Opin. Pharmacol. 8, 1-7 (2008).

17 Hopwood, Sir D. J. et al. Call to arms. Nat. Rev. Drug Disc. 6, 8-12 (2007).

18 Singh, S. B. \& Palaez, F. Biodiversity, chemical diversity and drug discovery. Progr. Drug Research 65, 143-174 (2008).

19 Clardy, J., Fischbach, M. A. \& Walsh, C. T. New antibiotics from bacterial natural products. Nat. Biotechnol. 24, 1541-1548 (2006).

20 Palaez, F. The historical delivery of antibiotics from microbial natural products - can history repeat? Biochem. Pharmacol. 71, 981-990 (2006).

21 Bérdy, J. in Are actinomycetes exhausted as a source of secondary metabolites? The Biology of Actinomycetes '94 Proc. 9th Int. Symp. Biology Actinomycetes (eds Debanov, V. G. et al.) 13-24 (Biotechnol. Acad., Moscow, Russia, 1995).

22 Harvey, A. L. Natural products as a screening resource. Curr. Opin. Chem. Biol. 11 , 480-484 (2007).
23 Simmons, T. L. et al. Biosynthetic origin of natural products isolated from marine microorganism-invertebrate assemblaages. Proc. NatI Acad. Sci. USA 105, 4587-4594 (2008).

$24 \mathrm{Hu}, \mathrm{G}$. P. et al. Statistical research on marine natural products based on data obtained berween 1985 and 2008. Mar. Drugs 9, 514-525 (2011).

$25 \mathrm{Liu}, \mathrm{X}$. Y. et al. Bioprospecting microbial natural product libraries from the marine environment for drug discovery. J. Antibiot. 63, 415-422 (2010).

26 Imhoff, J. F., Labes, A. \& Wiese, J. Bio-mining the microbial treasures of the ocean: new natural products. Biotechnol. Adv. 29, 468-482 (2011).

27 Kunsari, S. \& Spiteller, M. Are we ready for industrial production of bioactive plant secondary metabolites utilizing endophytes? Nat. Prod. Rep. 28, 1203-1207 (2011).

28 Crawford, J. \& Clardy, J. Bacterial symbionts and natural products. Chem. Comm. 47, 7559-7566 (2011).

$29 \mathrm{Yu}, \mathrm{H}$. S. et al. Recent development and future prospects of antimicrobial metabolites produced by endophytes. Microbiol. Res. 165, 437-449 (2010).

30 Simmons, L. T. et al. Biosynthetic origin of natural products isolated from marine microorganisms-invertebrate assemblages. Proc. Natl Acad. Sci. USA 105, 4587-4594 (2008).

31 Rath, C. M. et al. Meta-omic characterization of the marine invertebrate microbial consortium that produces the chemotherapeutic natural product ET-743, ACS. Chem. Biol. 61, 1244-1256 (2011).

32 Vályi-Nagy, T., Úri, J. \& Szilágyi, I. Primycin, a new antibiotic. Nature 174, 1105-1106 (1954).

33 Wilkinson, B. \& Micklefield, J. Mining and engineering natural-product biosynthetic pathways. Nature Chem. Biol. 3, 379-386 (2007).

34 Bode, H. B. \& Müller, R. The impact of bacterial genomics on natural product research. Angew. Chem. 44, 6828-6846 (2005).

35 Monagham, R. L. \& Barrett, J. F. Antibacterial drug discovery-Then., now and the genomic future. Biochem. Pharmacol. 71, 901-909 (2006).

36 Böttcher, T., Pitscheider, M. \& Sieber, S. A. Natural products and their biological targets: Proteomic and metabolomic labeling strategies. Angew. Chem. Int. Ed. 49, 2650-2698 (2010).

37 Olano, C., Lombó, F., Méndez, C. \& Salas, J. A. Improving production of bioactive secondary metabolites in actinomycetes by metabolic engineering. Metab. Eng. 281, 281-292 (2008).

38 Pickens, L. B., Tang, Y. \& Chooi, Y. H. Metabolic engineering for the production of natural products. Annu. Rev. Chem. Biomol. Engineering 2, 211-236 (2011).

39 Winter, J. M., Behnken, S. \& Hertweck, C. Genomic-inspired discovery of natural products. Curr. Opin. Chem. Biol. 15, 22-31 (2011).

40 Van Landen, s. G. \& Shen, B. Microbial genomics for the improvement of natural product discovery. Curr. Opin. Microbiol. 9, 252-260 (2006).

41 Singh, B. K. \& Macdonald, C. A. Drug discovery from uncultivable microorganisms. Drug. Discov. Today 15, 792-799 (2010).

42 Yim, G., Wang, H. H. \& Davies, J. The truth about antibiotics. Int. J. Med. Microbiol. 296, 163-170 (2006).

43 Davies, J. Are antibiotics naturally antibiotics? J. Ind. Microbiol. Biotechnol. 33, 496-499 (2006).

44 Bostian, M., McNitt, K. \& Bérdy, J. Antibiotic identification: a computerized data base system. J. Antibiot. 30, 633-634 (1977).

45 Berdy, J in Handbook of Antibiotic Compounds (Ed. Aszalos, A., Bostian, M. \& McNitt, K.) Vol. I to XIV/2 (CRP Press, Boca Raton, 1980-1987).

46 Bérdy, J in Dictionary of Natural Products (CRC Press, Boca Raton, 1988-1994).

47 Singh, R. K., Tiwari, S. P., Rai, A. K. \& Mohapatra, T. M. Cyanobacteria: an emerging source for drug discovery. J. Antibiot. 64, 401-412 (2011).

48 Bérdy, J. et al. Metabolites of gentamicin producing Micromonospora species, Part 1. Isolation and identification of metabolites. J. Antibiot. 30, 945-953 (1977).

49 Umezawa, H. in Enzyme Inhibitors of Microbial Origin (University of Tokyo Press, Tokyo, Japan, 1972).

50 Greenberg, E. P. Sociomicrobiology: A personal perspective on an emerging research area. Microbe Magazine (ASC (2010).

51 Linares, J. F., Gustaffson, I., Baquero, F. \& Martinez, J. L. Antibiotics as intermicrobial signaling agents instead of weapons. Proc. Natl Acad. Sci. USA 103, 19484-19489 (2006).

52 Williams, P. Quorum sensing, communication and cross-kingdom signalling in the bacterial world. Microbiology 153, 3923-3938 (2007).

53 Clardy, J. \& Walsh, C. Lessons from natural molecules. Nature 432, 829-837 (2004).

54 Anonymous. Antibiotics au naturel. Nat. Biotechnol. 24, 1494-1496 (2006).

55 Phillips, J. W. et al. Discovery of kibdelomycin a potent new class of bacterial type II topoisomerase inhibitor by chemical-genetic profiling in Staphylococcus aureus. Chem. Biol. 18, 955-965 (2011).

56 Hong, J. Role of natural product discovery in chemical biololgy. Curr. Opin. Chem. Biol. 15, 350-354 (2011)

57 Demain, A. L. From natural products discovery to commercialization: a success story. J. Ind. Microbiol. Biotechnol. 33, 486-495 (2006).

58 Harvey, A. L. Natural products as a screening resources. Curr. Opin. Chem. Biol. 11, 480-484 (2007).

59 Butler, M. S. Natural products to drugs: natural products-derived compounds in clinical trials. Nat. Prod. Res. 25, 475-516 (2008). 